\title{
SLAM using an Imaging Sonar for Partially Structured Underwater Environments
}

\author{
David Ribas, Pere Ridao \\ Dept. Electrònica, Informàtica i Automàtica \\ Universitat de Girona \\ Girona, Spain \\ \{dribas, pere\}@eia.udg.es
}

\author{
José Neira, Juan D. Tardós \\ Dept. Informática e Ingeniería de Sistemas \\ Universidad de Zaragoza \\ Zaragoza, Spain \\ \{jneira, tardos\}@unizar.es
}

\begin{abstract}
In this paper we describe a system for underwater navigation with AUVs in partially structured environments, such as dams, ports or marine platforms. An imaging sonar is used to obtain information about the location of planar structures present in such environments. This information is incorporated into a feature-based SLAM algorithm in a two step process: (1) the full $360^{\circ}$ sonar scan is undistorted (to compensate for vehicle motion), thresholded and segmented to determine which measurements correspond to planar environment features and which should be ignored; and (2) SLAM proceeds once the data association is obtained: both the vehicle motion and the measurements whose correct association has been previously determined are incorporated in the SLAM algorithm. This two step delayed SLAM process allows to robustly determine the feature and vehicle locations in the presence of large amounts of spurious or unrelated measurements that might correspond to boats, rocks, etc. Preliminary experiments show the viability of the proposed approach.
\end{abstract}

\section{INTRODUCTION}

The possibility of having truly autonomous vehicles heavily depends on their ability to build accurate models or maps of the environments they traverse, and to know their location in them. This has made this problem, known as Simultaneous Localization and Mapping (SLAM), the focus of a great deal of attention in recent years [1], [2], [3]. Many foreseen applications indoors, outdoors, on land, on air, and underwater will be possible once robust and efficient SLAM algorithms are available.

SLAM is currently considered solved for environments of limited size [4]. Algorithms based on both Extended Kalman Filters (EKFs) [5], [6], [7] and Particle Filter (PFs) [8], [9] can be used to map indoor and outdoor at least partially structured environments. Many of these systems use laser scanners, which provide precise 2D and 3D depth information. More recently, cameras are being used in SLAM systems due to their reduced cost and the possibility of obtaining texture information of the environment [10].

In some specially challenging environments and applications, such as underwater environments, it is not possible to use lasers, and there are also many difficulties in using cameras, among other issues due to lighting conditions. Sonar scanners have been used for a long time in underwater systems in general, and more recently for underwater SLAM systems [11], [12]. The range information provided by sonars is much

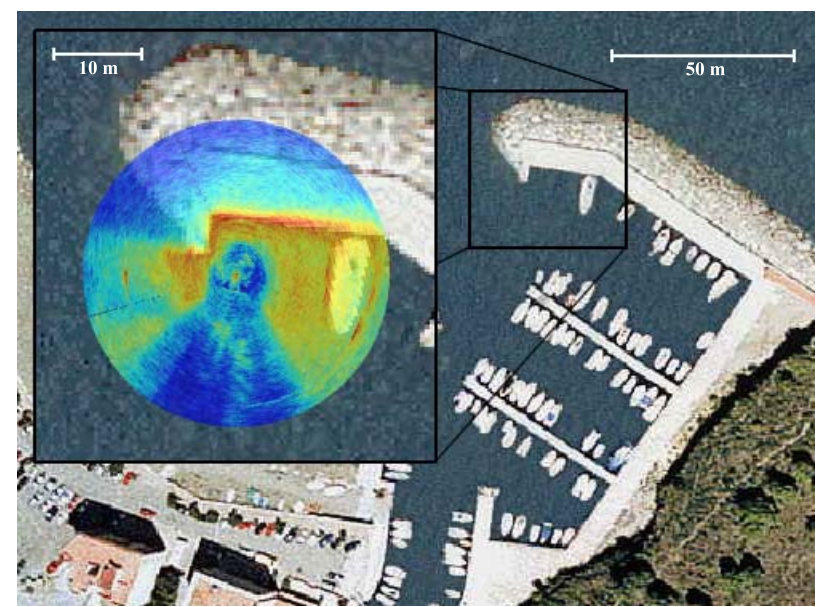

Fig. 1. Acoustic image superposed to a harbor satellite image

more difficult to interpret than laser scans because it is subject to more angular uncertainty. Also, spurious measurements are much more frequent. Underwater SLAM systems using sonars usually look for point features in the environment, which are very infrequent naturally in most underwater applications, and thus artificial landmarks have to be deployed.

In this paper we describe an underwater SLAM system for AUVs using an imaging sonar that builds a feature-based map of the environment, consisting in line features corresponding to planar structures in the environment. These types of structures are present in many environments of interest for AUVs such as dams, ports, or marine platforms (See Figure 1). They can be used to accurately determine the location of the vehicle in the environment, as it has been shown in indoor and outdoor urban environments. We use a mechanically scanning imaging sonar, a low cost acoustic sensor with an operating range of up to 100 meters which is usually used for obstacle detection. This imaging sonar provides a $360^{\circ}$ scan of the environment in 6.6 seconds. In contrast with laser scanners, this sensor provides 100 returns of different intensities for each beam, making more difficult the extraction of information regarding features such as stable planar structures. An additional difficulty lies in the fact that when mounted on an AUV, the resulting scan is distorted due to vehicle motion. To obtain an initial estimation 
of motion, the vehicle has a Doppler Velocity Log (DVL) that provides an estimation of velocity, as well as a compass and two inclinometers to provide absolute orientation.

SLAM cannot be carried out in the standard way because the information provided by the sensor in one direction is insufficient to carry out feature extraction and data association [6]. In this paper we describe the two step SLAM process that is carried out: (1) the full $360^{\circ}$ sonar scan is undistorted (to compensate for vehicle motion), thresholded and segmented to determine which measurements correspond to planar environment features and which should be ignored (section II); and (2) both the vehicle motion and the measurements whose correct association has been previously determined are incorporated in the SLAM algorithm (section III). Preliminary experiments (section IV) were carried out in our underwater laboratory to show the viability of the proposed approach.

\section{Segmentation And Feature Extraction}

Mechanically scanning sonars perform scans in a 2D plane by rotating a sonar beam through a series of small angle steps. For each emitted beam, distance vs. echo-amplitude data is returned forming an acoustic image of the surroundings (Figure 2). The scanning rate of these devices is really slow in comparison with multibeam sonars. For this reason, the vehicle movement along a complete scan usually induces important distortions in the acoustic image (See Figure 2(b)). Extracting features from this kind of images produces inaccuracies and yield to poor results. To cope with the slow scanning rate of the low cost imaging sonars, we propose a 2 step line extraction procedure. First, the trajectory of the vehicle is estimated at the same time that the acoustic beams are grabbed (described in section A). Then, when the position of each beam is known, the distortion induced by motion is compensated (Figure 2(c)). The segmentation procedure together with the Hough transform yield the lines present in the undistorted acoustic image as reported in section B.

\section{A. Trajectory estimation}

In order to be able to correct the image, whenever a new sonar beam is read, it is tagged with the current robot pose. A SonTek Argonaut DVL unit which includes a compass, 2 inclinometers and a depth sensor is used to estimate the robot pose (navigation problem). Sonar beams are read at $30 \mathrm{~Hz}$ while DVL readings arrive asynchronously at a frequency within $1.5 \mathrm{~Hz}$ interval. An EKF is used to estimate the 6DOF robot pose whenever a sonar beam is read. DVL readings are used asynchronously to update the filter. To reduce noise inherent to the DVL measurements, a simple 6DOF constant velocity kinematics model is used instead of a more conventional dead reckoning method. Since AUVs are commonly operated describing rectilinear transects at constant speed during survey missions, we believe that the proposed model is a simple but realistic way to describe the motion. Equations 1 and 2 show the state vector and the proposed state space model:

$$
\mathbf{x}_{R}^{B}=\left[\eta^{B}, \nu^{R}\right]^{T}
$$

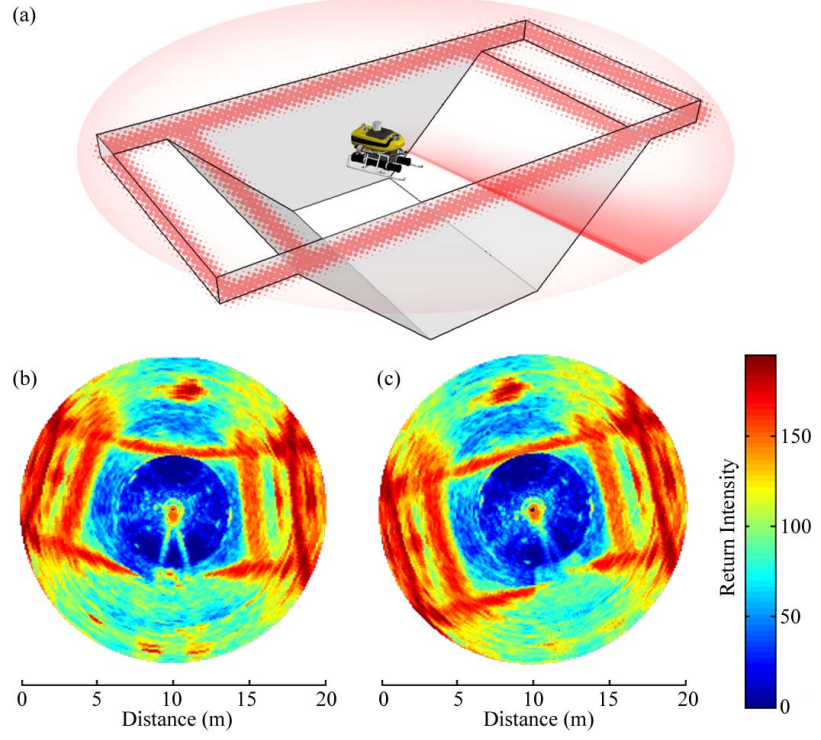

Fig. 2. (a) Schematic representation of the environment where the sonar data were gathered. The highlighted zones represent the expected sonar returns. Images generated from acoustic data, (b) distorted and (c) undistorted image through DVL integration

$$
\mathbf{x}_{R, k}^{B}=f\left(\mathbf{x}_{R, k-1}^{B}\right)=\left[\begin{array}{l}
\eta_{k}^{B} \\
\nu_{k}^{R}
\end{array}\right]=\left[\begin{array}{c}
\eta_{k-1}^{B}+J\left(\eta_{k-1}^{B}\right) \nu_{k-1}^{R} T \\
\nu_{k-1}^{R} T
\end{array}\right]
$$

with:

$$
\begin{gathered}
\eta^{B}=[x, y, z, \phi, \theta, \psi]^{T} ; \quad \nu^{R}=[u, v, w, p, q, r]^{T} \\
J(\eta)=\left[\begin{array}{cccccc}
c \psi c \theta & c \psi s \theta s \phi-s \psi c \phi & c \psi s \theta c \phi+s \psi s \phi & 0 & 0 & 0 \\
s \psi c \theta & s \phi s \psi s \theta+c \psi c \phi & s \psi s \theta c \phi-s \phi c \psi & 0 & 0 & 0 \\
-s \theta & c \theta s \phi & c \theta c \phi & 0 & 0 & 0 \\
0 & 0 & 0 & 1 & s \phi t \theta & c \phi t \theta \\
0 & 0 & 0 & 0 & c \phi & -s \phi \\
0 & 0 & 0 & 0 & s \phi / c \theta & c \phi / c \theta
\end{array}\right]
\end{gathered}
$$

where, as defined in [13], $\eta^{B}$ is the position and attitude vector referenced to a base frame $B$, and $\nu^{R}$ is the linear and angular velocity vector referenced to the robot coordinate frame $R$. The coordinate frame $B$ is oriented to the north. Hence, the compass measurements can be straight forward integrated. Although in this model the velocity is considered to be constant, in order to allow for slight movements, the velocity is modelled as the integral of a stationary white noise $\mathbf{v}_{k}$ with a diagonal $\mathbf{Q}$ in the order of magnitude of the maximum acceleration increment that the robot can experiment over a sample period.

$$
\begin{gathered}
\nu_{k}^{R}=\hat{\nu}_{k}^{R}+\mathbf{v}_{k} T \\
E\left[\mathbf{v}_{k}\right]=0 ; \quad E\left[\mathbf{v}_{k}, \mathbf{v}_{j}^{T}\right]=\delta_{k_{j}} \mathbf{Q}
\end{gathered}
$$

Hence, the acceleration noise is additive in the velocity (Equation 5) and propagates nonlinearly to the position. Finally, the model prediction and update is carried out as detailed below:

1) Prediction: The estimate of the state is obtained as:

$$
\hat{\mathbf{x}}_{R, k}^{B}=f\left(\hat{\mathbf{x}}_{R, k-1}^{B}\right)
$$


and its covariance matrix as:

$$
\mathbf{P}_{R, k}^{B}=\mathbf{F}_{R, k} \mathbf{P}_{R, k-1}^{B} \mathbf{F}_{R, k}^{T}+\mathbf{G}_{R, k} \mathbf{Q}_{k} \mathbf{G}_{R, k}^{T}
$$

where $\mathbf{F}_{R, k}$ and $\mathbf{G}_{R, k}$ are the Jacobian matrices of partial derivatives of the non-linear model function $f$ with respect to the state $\mathbf{x}_{R, k}^{B}$ and the noise $v_{k}$, respectively.

2) Update: The model prediction is updated by the standard Kalman filter equations each time a new DVL measurement is available:

$$
\mathbf{z}_{S, k}=\left[u_{b}, v_{b}, w_{b}, u_{w}, v_{w}, w_{w}, \phi_{i}, \theta_{i}, \psi_{c}, z_{\text {depth }}\right]^{T}
$$

where subindex $b$ stands for bottom tracking velocity, $w$ for through water velocity, $i$ for inclinometers and $c$ represents the compass. The measurement model is:

$$
\begin{aligned}
& \mathbf{z}_{S, k}=\mathbf{H}_{S, k} \mathbf{x}_{k \mid k-1}^{B}+\mathbf{w}_{k}
\end{aligned}
$$

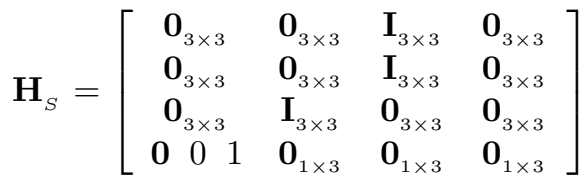

where $\mathbf{w}_{k}$ (measurement noise) is a zero-mean white noise:

$$
E\left[\mathbf{w}_{k}\right]=0 ; \quad E\left[\mathbf{w}_{k}, \mathbf{w}_{j}^{T}\right]=\delta_{k_{j}} \mathbf{R}
$$

Since the DVL sensor provides a status measurement for the bottom tracking and water velocity, depending on the quality of the measurements, different versions of the $\mathbf{H}$ matrix are used to fuse one (removing row 2), the other (removing row 1), or both readings (using the full matrix).

3) Trajectory Smoothing: Since the line extraction process is started when the full $360^{\circ}$ scan is completed, a smoothing procedure is used to provide the best estimate of the whole trajectory followed by the robot through the current scan. Hence, during the scanning, the state is augmented with each beam pose, and each DVL reading cause adjustments to the whole trajectory. Assuming an augmented state vector $\hat{\mathbf{x}}^{B}$, composed of both the actual vehicle state estimation at time $k$ and the rest of previously stored positions, the smoothing is performed by augmenting the state with a copy (or clone [14]) of the actual position estimate $\hat{\eta}_{k}^{B}$ and placing it immediately below the vehicle state $\hat{\mathbf{x}}_{k}^{B}$ :

$$
\hat{\mathbf{x}}_{k}^{B}=\left[\begin{array}{c}
\hat{\eta}_{k}^{B} \\
\hat{\nu}_{k}^{B} \\
\hat{\eta}_{k}^{B} \\
\vdots \\
\hat{\eta}_{1}^{B}
\end{array}\right] \quad \mathbf{P}_{k}^{B}=\left[\begin{array}{ccccc}
\mathbf{P}_{\eta, k}^{B} & \mathbf{P}_{\eta \nu, k}^{B} & \mathbf{P}_{\eta, k}^{B} & \ldots & \mathbf{P}_{\eta, k, 1}^{B} \\
\mathbf{P}_{\nu \eta, k}^{B} & \mathbf{P}_{\nu, k}^{B} & \mathbf{P}_{\nu \eta, k}^{B} & \ldots & \mathbf{P}_{\nu \eta, k, 1}^{B} \\
\mathbf{P}_{\eta, k}^{B} & \mathbf{P}_{\eta \nu, k}^{B} & \mathbf{P}_{\eta, k}^{B} & \ldots & \mathbf{P}_{\eta, k, 1}^{B} \\
\vdots & \vdots & \vdots & \ddots & \vdots \\
\mathbf{P}_{\eta, 1, k}^{B} & \mathbf{P}_{\eta \nu, 1, k}^{B} & \mathbf{P}_{\eta, k, 1}^{B} & \ldots & \mathbf{P}_{\eta, 1}^{B}
\end{array}\right]
$$

As a consequence of this state augmentation, the equations presented in Sections II-A.1 and II-A.2 need to be adapted as follows:

$$
\left[\begin{array}{c}
\hat{\mathbf{x}}_{R, k+1}^{B} \\
\hat{\eta}_{k}^{B} \\
\vdots \\
\hat{\eta}_{1}^{B}
\end{array}\right]=\left[\begin{array}{c}
f\left(\hat{\mathbf{x}}_{R, k}^{B}, 0\right) \\
\hat{\eta}_{k}^{B} \\
\vdots \\
\hat{\eta}_{1}^{B}
\end{array}\right]
$$

$$
\mathbf{F}_{k}=\left[\begin{array}{cc}
\mathbf{F}_{R, k} & \mathbf{0}_{12 \times 6 k} \\
\mathbf{0}_{6 k \times 12} & \mathbf{I}_{6 k \times 6 k}
\end{array}\right] \quad \mathbf{G}_{k}=\left[\begin{array}{c}
\mathbf{G}_{R, k} \\
0_{6 k \times 12}
\end{array}\right]
$$

Finally, at the correction step only $\mathbf{H}_{S}$ needs to be adapted by adding $6 k$ zero columns. Therefore, the cloning and smoothing continues until the end of the scan when we have in the state vector the best trajectory estimation. At the beginning of the next scan, the previously stored positions are removed before resuming the process.

\section{B. Line extraction}

Once an estimation of the vehicle trajectory through the sonar scan has been obtained, it is time to search for possible features. Since objects present in the environment appear as high echo-amplitude returns, a thresholding is applied to discard low echo returns which contain no significant information. Then, a search for local maximums is carried out for each beam. This reduces the number of considered measurements without appreciable loss in the accuracy of the features. Since we know the coordinates of each single echo return $\hat{\mathbf{x}}_{P}^{R}$ with respect to the vehicle reference $R$, the corrected position is computed using with the vehicle position after the cloning process (through the transformation-point feature composition operator [6]):

$$
\hat{\mathbf{x}}_{P}^{B}=\hat{\eta}_{R}^{B} \oplus \hat{\mathbf{x}}_{P}^{R}
$$

Figure 3(a) shows the resulting position of the thresholded sonar returns from a single scan.

For line extraction in this type of images, a Hough transform with a particular voting scheme has been used. When extracting lines from sonar data using voting schemes like Hough, authors [6] have proposed to vote to all the tangent lines to the sonar arc. In that case, the sonar beam had around $20-30^{\circ}$ of aperture. When dealing with our imaging sonar, which makes use of narrow horizontal beam ( $3^{\circ}$ of aperture) we observed that the narrow beams were able to detect walls whose surface was not tangent to the corresponding arc, but within an interval. For this reason, we propose to vote not only to the tangent lines but to the lines with a slope slightly higher or lower than the tangent ones. Since we are interested in line features, the Hough space is parameterized by $\rho^{B}$ and $\theta^{B}$. Then, each sonar return position vote on this discretized parametric space for all the candidate lines according to the above mentioned sonar model. In Figure 3(b) the resulting Hough votation space is shown. The selection among the candidate lines starts by rejecting the lines which had not received a minimum number of votes. Next, the most voted line from each cluster is selected. Finally we force the line to have a minimum density of points, filtering those whose points are scattered through line. At the end of the process, the pointto-line association has been established. The result is the set of detected lines together with the set of points belonging to them conveniently labelled. 


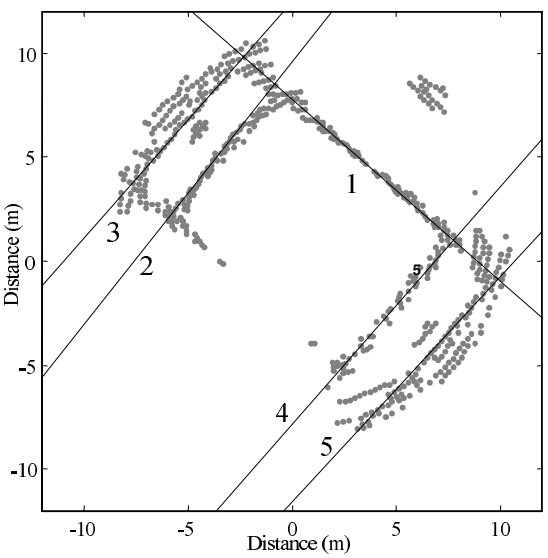

(a)

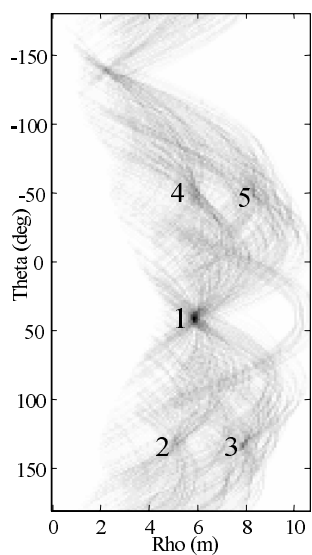

(b)
Fig. 3. Hough transform for line detection. (a) High echo-amplitude returns and the winning lines. (b)The obtained Hough voting space

\section{DELAYED SLAM}

Each time a scan is completed, the segmentation and feature extraction process provides a set of features and the correspondences with the measured points. This information is only available when the entire scan is processed. Therefore, the SLAM algorithm must run in a delayed manner

The stochastic map information is stored in the state vector $\mathbf{x}^{B}$ which contains the vehicle state $\mathbf{x}_{R}^{B}$, estimated as explained in Sections II-A.1 and II-A.2, as well as the line features conforming the map of the surroundings. Therefore, this state augmentation makes it necessary to adapt the equations in a similar way as reported in 14 and 15 . From this, the algorithm will be structured in two steps:

\section{A. Line feature estimation}

The process of the line estimation uses the information obtained from the feature extraction algorithm to initialize the lines in the map and then process the associated measurements to estimate the line uncertainty.

1) Initialization: At the beginning of a scan, the state is augmented with the previously detected lines $\hat{\mathbf{x}}_{L_{n}}^{B}$ :

$$
\hat{\mathbf{x}}_{0}^{B}=\left[\begin{array}{c}
\hat{\mathbf{x}}_{R}^{B} \\
\hat{\mathbf{x}}_{L_{1}}^{B} \\
\vdots \\
\hat{\mathbf{x}}_{L_{n}}^{B}
\end{array}\right] \quad \mathbf{P}_{0}^{B}=\left[\begin{array}{cccc}
\mathbf{P}_{R}^{B} & 0 & \ldots & 0 \\
0 & \mathbf{P}_{L_{1}}^{B} & \ldots & 0 \\
\vdots & \vdots & \ddots & \vdots \\
0 & 0 & \ldots & \mathbf{P}_{L_{n}}^{B}
\end{array}\right]
$$

Where $\hat{\mathbf{x}}_{L_{n}}^{B}=\left[\rho^{B}, \theta^{B}\right]^{T}$ and the line parameters $\rho^{B}$ and $\theta^{B}$ are the values obtained by the Hough transform. The line covariances $\mathbf{P}_{L_{n}, k}^{B}$ are initialized with a large uncertainty value as an uninformative prior.

2) Update: The vehicle movement is estimated as usual, but when a selected measurement happens it is introduced in the map as a point feature $\hat{\mathbf{x}}_{P_{m}}^{B}$ :

$$
\hat{\mathbf{x}}_{k}^{B}=\left[\begin{array}{c}
\hat{\mathbf{x}}_{R}^{B} \\
\vdots \\
\hat{\mathbf{x}}_{L_{n}}^{B} \\
\vdots \\
\hat{\mathbf{x}}_{P_{m}}^{B}
\end{array}\right]=\left[\begin{array}{c}
\hat{\mathbf{x}}_{R}^{B} \\
\vdots \\
\hat{\mathbf{x}}_{L_{n}}^{B} \\
\vdots \\
\hat{\eta}_{R}^{B} \oplus \hat{\mathbf{x}}_{P_{m}}^{R}
\end{array}\right]
$$

Then, it is necessary to update the estimated error covariance matrix:

$$
\begin{gathered}
\mathbf{P}_{k}^{B}=\mathbf{F}_{k} \mathbf{P}_{k}^{B} \mathbf{F}^{T}+\mathbf{G} \mathbf{P}_{P_{m}}^{R} \mathbf{G}^{T} \\
\mathbf{F}_{k}=\left[\begin{array}{cccc}
\mathbf{I} & 0 & \ldots & 0 \\
\vdots & \vdots & \ldots & \vdots \\
0 & 0 & \ldots & \mathbf{I} \\
\mathbf{J}_{1 \oplus} & 0 & \ldots & 0
\end{array}\right] \quad \mathbf{G}_{k}=\left[\begin{array}{c}
0 \\
\vdots \\
0 \\
\mathbf{J}_{2 \oplus}
\end{array}\right]
\end{gathered}
$$

where $\mathbf{J}_{1 \oplus}$ and $\mathbf{J}_{2 \oplus}$ are the Jacobians of the composition transformation [15] and $\mathbf{P}_{P_{m}}^{R}$ is the covariance of the point measured from the vehicle with the sonar. The same procedure is repeated for all the points.

3) Merge sonar returns: At the end of a scan, the state vector should contain the detected lines (with total uncertainty) and their corresponding sonar measurements. As the association between the points and the lines is perfectly known we can merge the information and thus, estimate the line state. Lets suppose that $\mathcal{H}_{P}$ is the hypothesis relating each sonar return with its associated line. Since both the features and the measurements belong to the state, we can represent the condition that the points correspond to each line by an ideal measurement equation without noise [16]:

$$
\mathbf{z}_{k}=h_{\mathcal{H}_{P}}\left(\mathbf{x}_{k}^{B}\right)=0
$$

Then, the state can be updated using a modified version of the EKF update equations [6], with $\mathbf{z}_{k}=0$ and covariance equal to zero. As $h_{\mathcal{H}_{P}}$ is the point to line distance nonlinear function it is necessary to linearize. The update is performed as:

$$
\begin{gathered}
\mathbf{K}_{\mathcal{H}_{P}}=\mathbf{P}_{k \mid k-1}^{B} \mathbf{H}_{\mathcal{H}_{P}}^{T}\left(\mathbf{H}_{\mathcal{H}_{P}} \mathbf{P}_{k \mid k-1}^{B} \mathbf{H}_{\mathcal{H}_{P}}^{T}\right)^{-1} \\
\hat{\mathbf{x}}_{k}^{B}=\hat{\mathbf{x}}_{k \mid k-1}^{B}-\mathbf{K}_{\mathcal{H}_{P}} h_{\mathcal{H}_{P}}\left(\hat{\mathbf{x}}_{k}^{B}\right) \\
\mathbf{P}_{k}^{B}=\left(\mathbf{I}-\mathbf{K}_{\mathcal{H}_{P}} \mathbf{H}_{\mathcal{H}_{P}}\right) \mathbf{P}_{k \mid k-1}^{B}
\end{gathered}
$$

After the update, the lines have been estimated using the information from the points. Hence, the lines are correlated with the vehicle pose and its uncertainty has been totally determined from the uncertainty of the corresponding points. Finally, the points are removed from the state vector.

\section{B. Matching lines between scans}

Every time a scan is completed, a set of lines is estimated and introduced into the stochastic map. Lines from different scans may correspond to the same object from the environment. They are thus susceptible to be associated and merged in order to correct the whole state. 


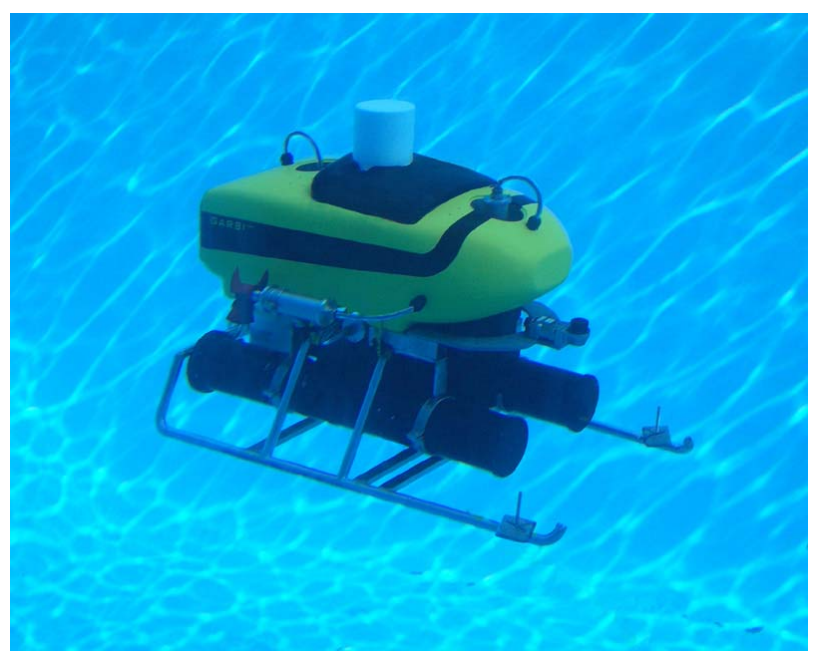

Fig. 4. The GARBI ${ }^{A U V}$

1) Compatibility test: To produce the update, an association hypothesis is needed. For this purpose, an individual compatibility test is performed at the end of each scan between each new line and all the lines previously in the map. Again, an ideal measurement equation without noise is used:

$$
\mathbf{z}_{k}=h_{\mathcal{H}_{k}}\left(\mathbf{x}_{k}^{B}\right)=0
$$

Taking into account that the Jacobian of the nonlinear function $h_{\mathcal{H}}$ is $\mathbf{H}_{\mathcal{H}}$, a compatibility test can be written as:

$$
D_{\mathcal{H}}^{2}=h_{\mathcal{H}_{k}}\left(\hat{\mathbf{x}}_{k}^{B}\right)^{T} \mathbf{H}_{\mathcal{H}_{k}} \mathbf{P}_{k}^{B} \mathbf{H}_{\mathcal{H}_{k}}^{T} h_{\mathcal{H}_{k}}\left(\hat{\mathbf{x}}_{k}^{B}\right)<\chi_{d, \alpha}^{2}
$$

Distance $D_{\mathcal{H}}^{2}$ is the Mahalanobis distance. The correspondence is accepted if the distance is less than $\chi_{d, \alpha}^{2}$, with $\alpha$ defined as the confidence level and $d=\operatorname{dim}\left(h_{\mathcal{H}}\right)$.

The Nearest Neighbor (NN) selection criterion determines that among the features that satisfy (23), the one with the smallest Mahalanobis distance is chosen and hypothesis $\mathcal{H}_{k}$ is accepted.

2) Merge compatible lines: Having an Hypothesis $\mathcal{H}_{k}$ relating compatible lines, an update of the stochastic map can be performed:

$$
\begin{gathered}
\mathbf{K}_{\mathcal{H}_{k}}=\mathbf{P}_{k \mid k-1}^{B} \mathbf{H}_{\mathcal{H}_{k}}^{T}\left(\mathbf{H}_{\mathcal{H}_{k}} \mathbf{P}_{k \mid k-1}^{B} \mathbf{H}_{\mathcal{H}_{k}}^{T}\right)^{-1} \\
\hat{\mathbf{x}}_{k}^{B}=\hat{\mathbf{x}}_{k \mid k-1}^{B}-\mathbf{K}_{\mathcal{H}_{k}} h_{\mathcal{H}_{k}}\left(\hat{\mathbf{x}}_{k}^{B}\right) \\
\mathbf{P}_{k}^{B}=\left(\mathbf{I}-\mathbf{K}_{\mathcal{H}_{k}} \mathbf{H}_{\mathcal{H}_{k}}\right) \mathbf{P}_{k \mid k-1}^{B}
\end{gathered}
$$

The lines estimated in the last scan which match with a feature already in the map can now be eliminated from the state.

\section{EXPERIMENTAL RESUlTS}

We carried out an experiment with the GARBI ${ }^{\mathrm{AUV}}$ (See Figure 4) in the water tank of the Underwater Robotics Research Center at the University of Girona (See Figure 5). The vehicle was equipped with a Miniking Imaging sonar from Tritech, a sensor designed for use in underwater applications
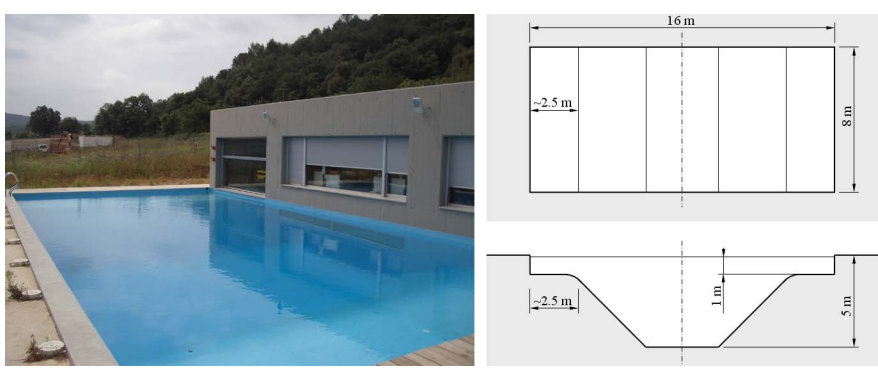

Fig. 5. Water tank at the Research Center on Underwater Robotics of the University of Girona

like obstacle avoidance and target tracking. It can perform scans in a 2D plane by rotating a fan-shaped sonar beam of $3^{\circ}$ of horizontal beamwidth and $40^{\circ}$ of vertical beamwidth. During the experiment, the sensor was set up to work within a range of 10 meters, capturing a sonar return every 0.1 meters (100 measurements per beam). Its scanning rate was set to the maximum (around 6 seconds per a $360^{\circ}$ scan).

In order to estimate the vehicle movement an Argonaut DVL from Sontek was used. The DVL is a sensor specially designed for ROV/AUV applications which measures ocean currents and vehicle speed over ground, by means of the Doppler shift effect, and altimetry. Moreover, the unit is also equipped with a compass/tilt sensor which permits to recollect attitude data, a pressure sensor to estimate the depth and a temperature sensor for sound speed calculations.

The GARBI ${ }^{\mathrm{AUV}}$ carried out a guided trajectory of around 42 meters, consisting on several loops; 161 complete sonar scans were taken.

The final map obtained is shown in Figure 6. The estimated lines are shown in light gray, represented within a 95\% confidence level, while the tank contour had been highlighted for easier identification. For comparison purposes the trajectory estimated using dead reckoning of the DVL measurements (blue dash-dotted line) is represented together with the SLAM estimated one (black line). As it can be seen, the dead reckoning trajectory has an important drift, which is controlled using the proposed SLAM algorithm. As the tank dimensions are known, they can be used as a ground truth for evaluation of the resulting map. Figure 7 shows the error plots for the estimation of the length of the four boundary walls. We can clearly see that errors remain inside the $2 \sigma$ bounds most of the time.

\section{CONClusion}

Most of the previous work in underwater SLAM has been focused on the use of point features. The main contribution of this work consists on carrying out underwater SLAM detecting lines using sonar imagery in a partially structured environment like harbors or dams. We propose a method to correct the motion that induces distortion commonly present in the sonar scans gathered with low cost, slow, mechanically scanning sonar. Trajectory smoothing by cloning along a sonar scan is carried out to the best estimate of the robot trajectory at the 
end of the scan. The smoothing improves the results when the velocity measurements coming from the DVL are very noisy or include outliers. For an imaging sonar which provides echo intensities along the sonar beam, voting to an interval of lines enclosing the lines tangent to the sonar arc, has probed to be very important for line detection. One of the weakest points of our current implementation stands for the low frequency of the DVL measurements. This is particularly important for the heading, forcing us to use a pessimistic error model for the compass. Using a higher frequency compass, would allow us to increment the operation velocity of the robot, specially the yaw rate, without compromising the correction of the distortion.

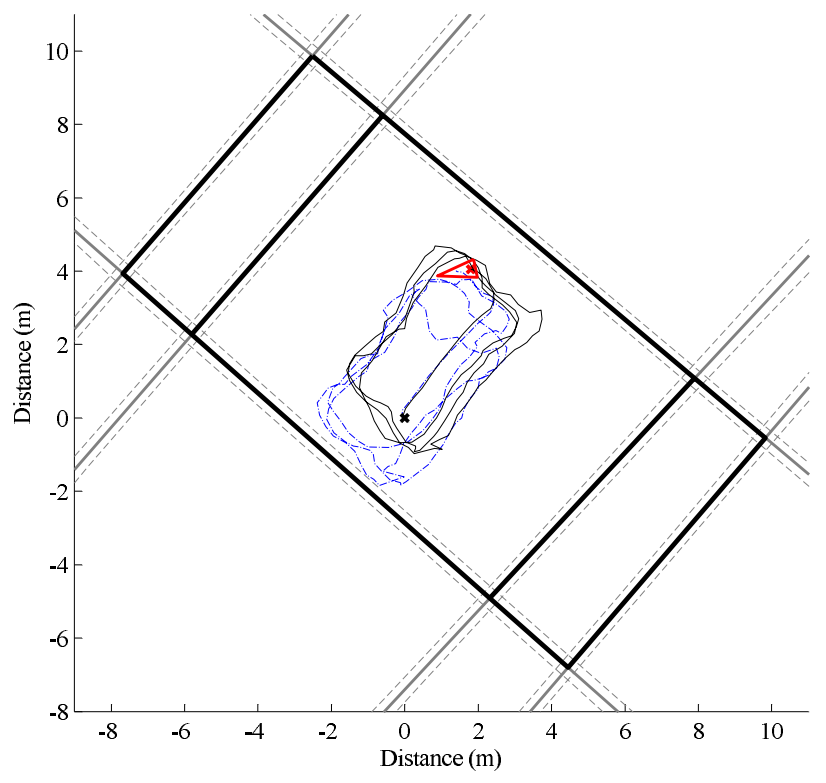

Fig. 6. Global map obtained using sonar and DVL together
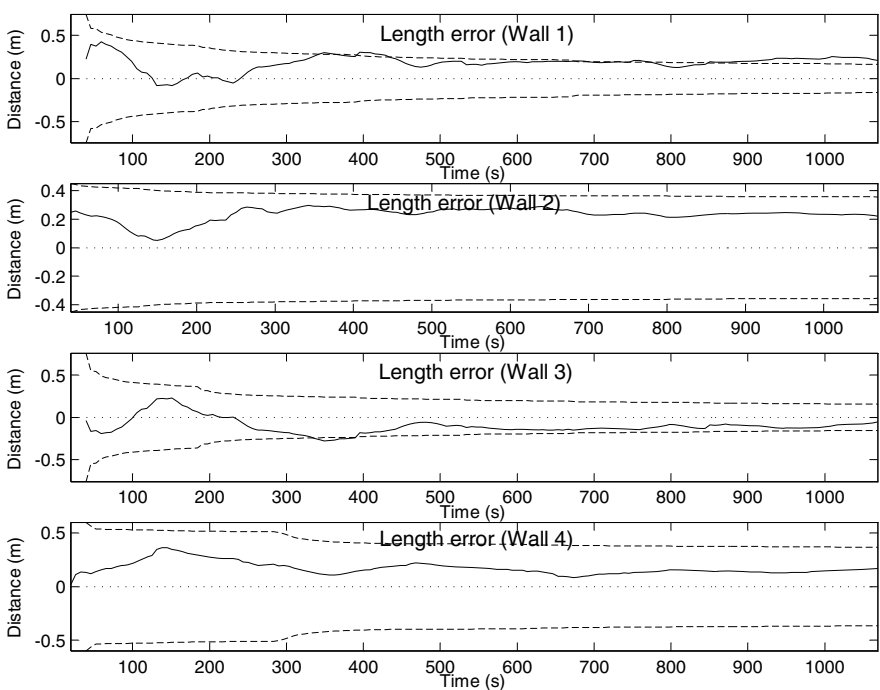

Fig. 7. Errors for the estimated length of the four boundary walls

\section{ACKNOWLEDGMENT}

This research has been funded in part by the Dirección General de Investigación of Spain under projects DPI200307986 and DPI2005-09001-C03-01.

\section{REFERENCES}

[1] J. Leonard and H. Durrant-Whyte, Directed Sonar Sensing for Mobile Robot Navigation. London: Kluwer Academic Publishers, 1992.

[2] J. A. Castellanos and J. D. Tardós, Mobile Robot Localization and Map Building: A Multisensor Fusion Approach. Boston, Mass.: Kluwer Academic Publishers, 1999.

[3] S. Thrun, W. Burgard, and D. Fox, Probabilistic Robotics. The MIT Press, 2005

[4] M. W. M. G. Dissanayake, P. Newman, S. Clark, H. F. Durrant-Whyte, and M. Csorba, "A solution to the simultaneous localization and map building (SLAM) problem," IEEE Trans. on Robotics and Automation, vol. 17 , no. 3, pp. 229-241, 2001.

[5] J. A. Castellanos, J. M. M. Montiel, J. Neira, and J. D. Tardós, "The SPmap: A probabilistic framework for simultaneous localization and map building," IEEE Trans. Robot. Automat., vol. 15, no. 5, pp. 948953, 1999.

[6] J. Tardós, J. Neira, P. Newman, and J. Leonard, "Robust mapping and localization in indoor environments using sonar data," Int. J. Robotics Research, vol. 21, no. 4, pp. 311-330, 2002.

[7] S. Thrun, Y. Liu, D. Koller, A. Y. Ng, Z. Ghahramani, and H. DurrantWhyte, "Simultaneous Localization and Mapping with Sparse Extended Information Filters," The International Journal of Robotics Research, vol. 23, no. 7-8, pp. 693-716, 2004.

[8] M. Montemerlo, S. Thrun, D. Koller, and B. Wegbreit, "FastSLAM: A factored solution to the simultaneous localization and mapping problem," in Proceedings of the AAAI National Conference on Artificial Intelligence. Edmonton, Canada: AAAI, 2002.

[9] S. Thrun, "Particle filters in robotics," in Proceedings of Uncertainty in AI (UAI), 2002.

[10] A. Davison, "Real-time simultaneous localisation and mapping with a single camera," Oct. 2003.

[11] J. J. Leonard, R. Carpenter, and H. J. S. Feder, "Stochatic mapping using forward look sonar," Robotica, vol. 19, pp. 467-480, 2001.

[12] I. Tena, S. de Raucourt, Y. Petillot, and D. Lane, "Concurrent mapping and localization using sidescan sonar," IEEE Journal of Oceanic Engineering, vol. 29, no. 2, pp. 442-456, April 2004.

[13] T. Fossen, Guidance and Control of Ocean Vehicles. John Wiley \& Sons Ltd., 1994.

[14] S. Roumeliotis and J. Burdick, "Stochastic cloning: a generalized framework for processing relative state measurements," in Proceedings. ICRA '02. IEEE International Conference on Robotics and Automation, vol. 2, 2002, pp. 1788-1795.

[15] R. Smith, M. Self, and P. Cheeseman, "Estimating uncertain spatial relationships in robotics," in Autonomous robot vehicles. SpringerVerlag New York, Inc., 1990, pp. 167-193.

[16] J. Castellanos and J. Tardós, Mobile Robot Localization and Map Building: A Multisensor Fusion Approach. Kluwer Academic Publishers, Boston, 1999. 